\title{
Interactive comment on "Laboratory study of non-linear wave-wave interactions of extreme focused waves in the nearshore zone" by Iskander Abroug et al.
}

Iskander Abroug et al.

iskander.abroug@unicaen.fr

Received and published: 8 September 2020

The authors would like to thank the reviewer for his thoughtful and useful comments on our paper. We have considered all the suggestions. Our point-by-point responses (R) to comments and questions $(\mathrm{Q})$ are detailed below:

Q1:

The paper under review is devoted to the experimental study of nonlinear interactions in the focused wave packet by using the wavelet bi-spectral analysis. This experiment continues a series of studies done by these authors with the investigation the nonlinear

Printer-friendly version

Discussion paper 
dispersive mechanism of the rogue wave formation. In their experiments, the authors used the wave trains with various spectra (Pierson-Moskowitz and JONSWAP) in intermediate depth. Strong nonlinear effects are observed on the sloping beach as it is expected. Nonlinear energy transfer in the high-frequency region is analyzed. In fact, such processes have been actively studied earlier, and, perhaps, the novel moment here is the demonstration of nonlinear effects through the bi-spectral analysis. To add to this, I would like the authors to formulate the obtained results in Conclusion better underlying their difference from the known results.

\section{R1:}

We would like to thank the reviewer for his remark. We tried to formulate and synthesise the obtained results in order to distinguish similarities and differences from the known results. Here is the conclusion. Redundant sentences have been deleted as well.

An experimental approach is proposed for determining the non-linear wave-wave interactions, which accompany the propagation of large amplitude wave trains, that might cause damage to coastal zones, marine structures and navigation vessels. We investigate seven focused wave trains derived from JONSWAP ( $\gamma=3.3$ or 7$)$ and PiersonMoskowitz spectra propagating from intermediate water depth to the inner surf zone. The results presented in this study extend the parameter range of observations of triad interactions. The experimental conditions were selected based on two parameters: the wave steepness and the spectrum type. The present data were collected in intermediate water with a kph0 varying between 0.92 and 0.79 . A typical wave train consists of a large number of waves interacting with one another. Wavelet-based bicoherence is used to investigate the phase coupling between frequency components of short time series. Some consequences of non-linear transfer are briefly discussed; in particular the role played by non-linear interactions in shaping the high frequency part of the spectrum, the relative contribution of each harmonic and the downshifting of the peak spectrum demonstrated in previous studies. Note that our experimental study is different from previous experiments (Dong et al., 2008; Ma et al., 2010) regarding the slope

Printer-friendly version

Discussion paper
Interactive

comment

\section{2}


geometry and most importantly, the use of three different spectral types.

Along the flat bottom $(4 \mathrm{~m}<\mathrm{x}<9.5 \mathrm{~m}$ ), one might assume that the influence of triad interactions is very weak for the three considered spectra. The bispectral analysis of the data shows that as the waves propagate along the flat bottom, the magnitude of the bicoherence increases slightly (between 0

Interactive

comment

When the wave train reaches the slope $(9.5 \mathrm{~m}<\mathrm{x}<\mathrm{xb})$, wave-wave interactions among high order harmonics increase rapidly and reach the maximum degree in the breaking/focus location. In line with previous studies (Elsayed, 2006; Dong et al., 2008; Ma et al., 2010), strong nonlinear interactions were predominantly observed in the shallower region. The analysis showed a gradual broadening of the bicoherence spectrum, which is in accordance with previous studies who demonstrated that the energy is transferred mainly to high frequencies regions (Tian et al. 2011; Abroug et al., 2020). This is partly due to significant spectral transformations which are more important during the shoaling process. Particularly, this analysis showed a considerable contribution of 2 nd and 3rd harmonics for unidirectional steep wave trains and the spectral components at the second harmonic $2 \mathrm{fp}$ have increased substantially (6 times its initial value). The bispectral analysis results show that the wave non-linearity S0 plays an important role in the increasing trend of phase coupling, which is more important for wave trains having strong non-linearities. This last finding agrees well with the conclusions made by Ma et al. (2010).

An innovative aspect of this paper is presenting wavelet-based bispectral analysis for highly non-linear intermediate water waves with different spectral types. If we compare the three spectra, we can see that all nonlinear interactions on the flat bottom $(x<$ $9.5 \mathrm{~m}$ ) are weak $\left(\mathrm{b}^{2}<0.15\right)$ in the case of wide spectrum wave trains (Tests 2 and 3 Fig. 11). However, in the case of narrower spectra, more frequencies (e.g. fp, $2 \mathrm{fp}$ and $3 \mathrm{fp}$ ) are implicated in the focusing process (Tests 4, 5, 6 and 7 Fig. 11) and the corresponding phase coupling is higher $\left(b^{2}>0.2\right)$. This finding is in agreement with the stable behavior of wide spectrum wave trains, which was demonstrated experimentally

Printer-friendly version Discussion paper (c) (i) 
in Abroug et al. (2019) and Stansberg (1994). In intermediate water depth $(0.79<\mathrm{kph}$ $<0.92$ ), wide spectrum harmonics ( $\mathrm{fp}, 2 \mathrm{fp}, 3 \mathrm{fp} .$. ) are less implicated in the focusing process compared to narrow spectrum harmonics. In shallow water regions $(9.5 \mathrm{~m}$ $<x<x b)$ and after breaking $(x b<x)$, the spatial evolution of the phase coupling is qualitatively similar for the three spectra.

The results obtained in this study show important features in wave-wave interactions during the propagation of focused waves. This study strengthens the usefulness of wavelet-based analysis in detecting features that are hidden in a Fourier-based analysis, and in explaining a number of phenomena, such as the process leading to wave breaking and the energy transfer between wave components. Nevertheless, in order to confirm the use of wavelet-based bicoherence for more realistic 3D studies with structures, efforts should be made to expand this study for example by investigating greater water depths, higher steepness and wider spectra. Furthermore, the observed evolution of bicoherence for focused waves should be compared to that of waves with similar steepness and bandwidth but with initial random distribution of phase. In other words, efforts should be made to identify and quantify the phase coupling differences between focusing wave trains and non-focusing waves. Information concerning the phase coherence can be obtained by calculating the biphase parameter ( $\beta$ (a1, a2), Ma et al., 2010). It would be interesting to quantitatively measure the deviation of biphase values between primary waves/higher harmonics and to analyse their spatial evolution through different spectra to distinguish differences. Finally, a detailed study of how bound energy at harmonics would be influenced by quadruplet interactions should be performed.

Q2: Equations (2) and (3) are written inaccurately. Function (3) does not contain tau and the parameter a.

Printer-friendly version

Discussion paper

We would like to thank the reviewer for this comment. I added Eq 4, which contains the

Interactive

comment

\section{R2:}


two parameters tau and a, in order to make Eq 3 more explicit (Line 130). Equation 4 has been added between the continuous wavelet transform WT $(a, \tau)$ function and the Morlet wavelet function.

$\left.\psi(a, \tau)(\mathrm{t})=|\mathrm{a}|^{(}-0.5\right) \psi((\mathrm{t}-\tau) / \mathrm{a})$

Q3:

For the wave focusing, it is necessary to vary the local frequency on the specific law for intermediate depth. I do not understand which formula for the local frequency versus time has been used. Perhaps, by using the optimal law, the focusing can occur on the flat bottom. If there is no specific focusing, there is an interference. Moreover, it should be reflected in the title.

\section{R3:}

It is an interesting comment and maybe the methodology of generation needs more clarifications from the authors. Here is the explanation, which was added to the manuscript.

Line (85-89)

The linear NewWave theory (Tromans et al. (1991)), which is able to generate targeted waves at a prescribed location and time by combining sinusoidal components of different frequencies, is used as input for the generated focused wave trains. This theory was validated at deep water locations, at intermediate water depth locations (Taylor and Williams (2004)) and at coastal regions (Whittaker et al. (2016); for kh < 0.5). In NewWave theory, the expected shape of a wave train is the autocorrelation function (Fourier Transform of the spectral density).

Line (100-111)

Using linear NewWave theory, the free surface elevation of a wave train at a distance $x$ from the wavemaker can be written as follows: 
$\eta(\mathrm{x}, \mathrm{t})=\sum_{(} i=1_{N} a_{i} \cos \left[k_{i}\left(x-x_{0}\right)-\omega_{i}\left(t-t_{0}\right)\right](1) a_{i}=A_{0}\left(S\left(f_{i}\right) \Delta \mathrm{f}\right) /\left(\sum_{(} i=1_{N} S\left(f_{i}\right) \Delta \mathrm{fãA}\right.$ Å $)$ (2)

where ai (Eq. (2)) is the amplitude of each component, i varies from 1 to $\mathrm{N}$ (number of waves), $\mathrm{x} 0$ and to denote respectively the predefined focal location and focal time, $\mathrm{ki}=\omega \mathrm{i} / \mathrm{gtanh}(\mathrm{kih})$ is the wavenumber, $\omega \mathrm{i}=2 \pi \mathrm{fi}$ is the angular frequency, $\mathrm{h}$ is the water depth, $\mathrm{A} 0$ represents the theoretical linear crest amplitude of the wave train, $\mathbf{S}(\mathrm{fi})$ is the spectral density and $\Delta \mathbf{f}=\left(\mathbf{f}_{m} a x-f_{m} i n\right) /(N-$ 1)isthe frequencystep.Thewavegroupisgeneratedwithagivenlinearfocusposition ( $x=$ $12 m$ fromthewavemaker)basedonlinear focusinginaconstantwaterdepth.

So to answer your question, yes, by modifying $x<9.5 \mathrm{~m}$ in the EDL Software, we can obtain a focusing on the flat bottom.

JONSWAP and Pierson-Moskowitz are the two spectra used to represent the sea state. All generated waves are crested focused waves, i.e. the phase angle of the wave group within its envelope at the focus position is equal to zero.

Please also note the supplement to this comment:

https://nhess.copernicus.org/preprints/nhess-2020-209/nhess-2020-209-AC1-

supplement.pdf

Interactive comment on Nat. Hazards Earth Syst. Sci. Discuss., https://doi.org/10.5194/nhess2020-209, 2020. 